Supporting Information

\title{
Morphology-controlled synthesis of colloidal polyphenol particles from aqueous solutions of tannic acid
}

Tero Kämäräinen, ${ }^{\dagger}$ Mariko Ago, ${ }^{\dagger}$ Luiz G. Greca, ${ }^{\dagger}$ Blaise L. Tardy, ${ }^{\dagger}$ Markus Müllner, ${ }^{\ddagger}$ Leena-Sisko Johansson ${ }^{\dagger}$ and Orlando J. Rojas ${ }^{*,+, \S}$

${ }^{\dagger}$ Department of Bioproducts and Biosystems, Aalto University, Vuorimiehentie 1, Espoo, P.O. Box 16300, FI-00076 Aalto, Finland.

${ }^{\ddagger}$ Key Centre for Polymers and Colloids, The University of Sydney, F11, Eastern Avenue, NSW 2006, Australia.

${ }^{\S}$ Department of Applied Physics, Aalto University, Puumiehenkuja 2, Espoo, P.O. Box 13500, Fl-00076 Aalto, Finland.

*Corresponding author: orlando.rojas@aalto.fi

\section{Table of Contents}

Experimental section

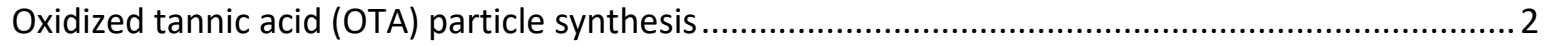

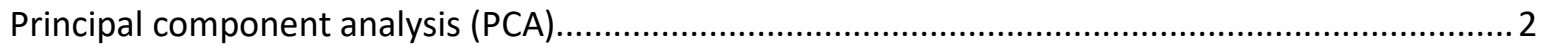

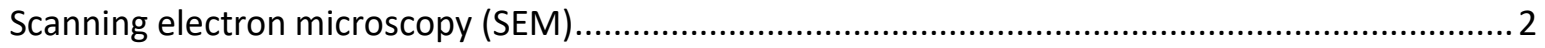

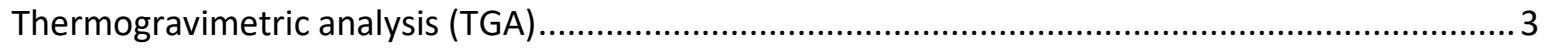

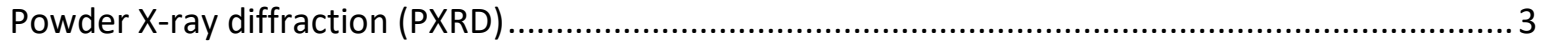

Fourier-transform infrared spectroscopy (FTIR) ......................................................................

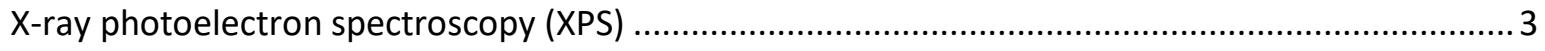

Matrix-assisted laser desorption/ionization mass spectrometry (MALDI) ...................................... 3

${ }^{13} \mathrm{C}$-Nuclear magnetic resonance spectroscopy with cross-polarization and magic-angle spinning

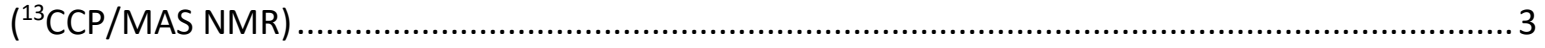

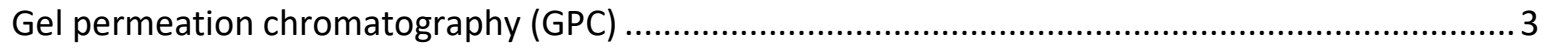

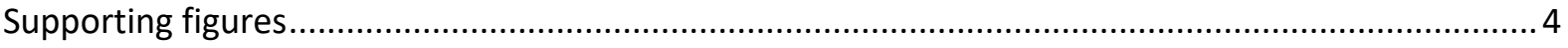

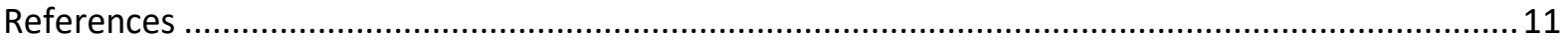




\section{Experimental section}

\section{Oxidized tannic acid (OTA) particle synthesis}

The reactions were carried out under shaking (orbital shaker) in glass vessels covered with perforated Parafilm to ensure continuous oxygenation of the solution. The $\mathrm{pH}$ of an aqueous tannic acid (1701.20 $\mathrm{g} / \mathrm{mol}$, Sigma-Aldrich) solution was adjusted to $7.8,9$ and 11 with either $1 \mathrm{M} \mathrm{KOH}, 1 \mathrm{M} \mathrm{LiOH}, 1 \mathrm{M}$ $\mathrm{NaOH}, 0.5 \mathrm{M} \mathrm{Na}_{3} \mathrm{PO}_{4}$ or $25 \% \mathrm{NH}_{4} \mathrm{OH}$. All chemicals were reagent grade and purchased from SigmaAldrich. The pH was measured and precipitates were collected after ca. $14 \mathrm{~h}$ or 1 week.

\section{Principal component analysis (PCA)}

The PCA was conducted using Matlab R2018a (MathWorks) for data gathered from precipitates produced from $2 \mathrm{wt} \%$ TA solutions, excluding dumbbell structures, by examining correlations in the data relative to the initial solution $\mathrm{pH}$, base conjugate acid $\mathrm{p} K_{\mathrm{a}}$, base counter cation ionic radius $(R)$, total yield $(Y)$ and characteristic dimension ratio $d_{2} / d_{1}$ and $d_{3} / d_{2}$ utilizing the mean particle size $d_{1}, d_{2}$ and $d_{3}$ (in descending size order) measured from SEM images for 10-30 particles using Image ${ }^{1}$ (Table S1). The data set was normalized along each dimension to zero mean and unit variance prior to the PCA. The analysis was narrowed to principal components (PCs) with an eigenvalue $>1$, accounting around $81 \%$ of the total variance (see Fig. S7). The selected PCs were then varimax rotated to aid the interpretation. $^{2}$

Table S1: Data utilized in the principal component analysis. Base counter cation radius $(R)$, base $\mathrm{p} K_{\mathrm{b}}$, base conjugate acid $\mathrm{p} K_{\mathrm{a}}$, initial solution $\mathrm{pH}$, particle characteristic dimensions in descending order $\left(d_{1}\right.$, $d_{2}, d_{3} \pm$ standard deviation) and total precipitate yield $(Y)$.

\begin{tabular}{lrrrrrrrr}
\hline Base & $R(\AA)^{3}$ & $\mathrm{p} K_{\mathrm{b}}$ & $\mathrm{p} K_{\mathrm{a}}$ & $\mathrm{pH}$ & $d_{1}(\mu \mathrm{m})$ & $d_{2}(\mu \mathrm{m})$ & $d_{3}(\mu \mathrm{m})$ & $Y(\%)$ \\
\hline & & & & 7.8 & $1.0 \pm 0.3$ & $0.6 \pm 0.2$ & $0.20 \pm 0.04$ & 13 \\
$\mathrm{KOH}$ & 1.33 & $-0.9^{4}$ & 14.9 & 9 & $1.6 \pm 0.5$ & $0.25 \pm 0.05$ & $0.25 \pm 0.05$ & 34 \\
& & & & 11 & $20 \pm 9$ & $0.43 \pm 0.02$ & $0.43 \pm 0.02$ & 47 \\
\hline & & & & 7.8 & $1.0 \pm 0.2$ & $0.6 \pm 0.1$ & $0.2 \pm 0.1$ & 7 \\
$\mathrm{NaOH}$ & 0.97 & $-0.8^{3}$ & 14.8 & 9 & $4 \pm 2$ & $1.8 \pm 0.8$ & $0.14 \pm 0.03$ & 24 \\
& & & & 11 & $12 \pm 3$ & $2.1 \pm 0.6$ & $0.2 \pm 0.1$ & 35 \\
\hline $\mathrm{LiOH}$ & 0.68 & $0.2^{3}$ & 13.8 & 9 & $5 \pm 4$ & $1.1 \pm 0.6$ & $0.8 \pm 0.4$ & 64 \\
& & & & 11 & $9 \pm 3$ & $1.9 \pm 0.3$ & $1.4 \pm 0.3$ & 28 \\
\hline \multirow{2}{*}{$\mathrm{Na}_{3} \mathrm{PO}_{4}$} & 0.97 & $2.23^{5}$ & 11.77 & 7.8 & $1.1 \pm 0.4$ & $0.7 \pm 0.3$ & $0.3 \pm 0.1$ & 24 \\
\hline $\mathrm{NH}_{4} \mathrm{OH}$ & 1.43 & $4.75^{3}$ & 9.25 & 11 & $4.1 \pm 0.9$ & $0.5 \pm 0.1$ & $0.5 \pm 0.1$ & 14 \\
\hline \hline
\end{tabular}

\section{Scanning electron microscopy (SEM)}

The oxidized tannic acid precipitates were imaged using a field-emission scanning electron microscope (Sigma VP, Zeiss, Germany) operating at $1.5 \mathrm{kV}$. The samples were prepared from aqueous dispersions on silicon wafers. After blotting the suspension on a silicon chip, the coated sample was sputtered with a $4 \mathrm{~nm}$ film of palladium/gold alloy. 


\section{Thermogravimetric analysis (TGA)}

TGA measurements were conducted with Q500 thermogravimetric analyzer (TA instruments, US) under $\mathrm{N}_{2}$ atmosphere using a $10^{\circ} \mathrm{C} / \mathrm{min}$ heating rate.

\section{Powder X-ray diffraction (PXRD)}

PXRD measurements were carried out using Rigaku SmartLab X-ray diffractometer equipped with a $\mathrm{Cu}$ $\mathrm{K} \alpha, \beta \mathrm{X}$-ray source. The scans were conducted over $2 \theta$ range $3-70^{\circ}$ with a scan rate of $15^{\circ} \mathrm{s}^{-1}$ and $0.01^{\circ}$ step size. The sample was neutralized and oven-dried at $80^{\circ} \mathrm{C}$ prior to characterization.

\section{Fourier-transform infrared spectroscopy (FTIR)}

FTIR spectra were obtained with Nicolet 380 FT-IR infrared spectrometer between $400-4000 \mathrm{~cm}^{-1}$ at $1.9 \mathrm{~cm}^{-1}$ resolution using 32 scans.

\section{$X$-ray photoelectron spectroscopy (XPS)}

The XPS spectra were obtained using AXIS Ultra electron spectrometer (Kratos Analytical, UK) with a $100 \mathrm{~W}$ mono $\mathrm{Al}-\mathrm{K} \alpha$ source and a charge neutralizer. Survey $\mathrm{C} 1 \mathrm{~s}$ and $\mathrm{O} 1 \mathrm{~s}$ spectra were acquired from three locations. The survey spectra were recorded with a pass energy of $80 \mathrm{eV}$ and peaks appearing at binding energies 285, 496, 533 and $1072 \mathrm{eV}$ were assigned to C1s, Na Auger, O1s and Na1s photoelectrons, respectively. ${ }^{6}$

\section{Matrix-assisted laser desorption/ionization mass spectrometry (MALDI)}

The mass spectra were acquired with Bruker ultrafleXtreme MALDI-TOF spectrometer. The prepared samples were diluted with milli-Q water 1000 or 10000 -fold. As matrix 2,5-dihydroxybenzoic acid $(154.12 \mathrm{~g} / \mathrm{mol})$ was used. The solutions of the sample and the matrix were mixed in equal amounts and $0.5 \mu \mathrm{L}$ of the resulting solution were applied on the MALDI target plate. The target plate was dried at ambient temperature in a dust-free environment and was introduced into the spectrometer.

\section{${ }^{13} \mathrm{C}$-Nuclear magnetic resonance spectroscopy with cross-polarization and magic-angle spinning (13 CCP/MAS NMR) \\ ${ }^{13} \mathrm{CCP} / \mathrm{MAS}$ NMR spectra of the dried OTA particles were measured using Bruker AVANCE III 400 spectrometer operating at $303 \mathrm{~K}$, spinning rate of $8 \mathrm{kHz}$ and frequency of $100 \mathrm{MHz}$. The sample was packed into zirconia rotors with a $4 \mathrm{~mm}$ diameter. Number of scans was 5000 .}

\section{Gel permeation chromatography (GPC)}

GPC measurements were performed on an UFLC Shimadzu Prominence system with Phenoge ${ }^{\mathrm{TM}}$ columns ( $5 \mu \mathrm{m}, 104 \AA$ and $105 \AA$ ) (eluent: $N, N$-dimethylacetamide containing BHT/LiBr at 0.05/0.03 wt\%) at a flow rate of $1 \mathrm{~mL} / \mathrm{min}$ at $50^{\circ} \mathrm{C}$. Samples were dissolved in $0.5 \mathrm{~mL}$ eluent and passed through a $220 \mathrm{~nm}$ Nylon filter prior to injection. The system was calibrated with near monodisperse PMMA standards from Polymer Standards Service (PSS, Germany). 


\section{Supporting figures}

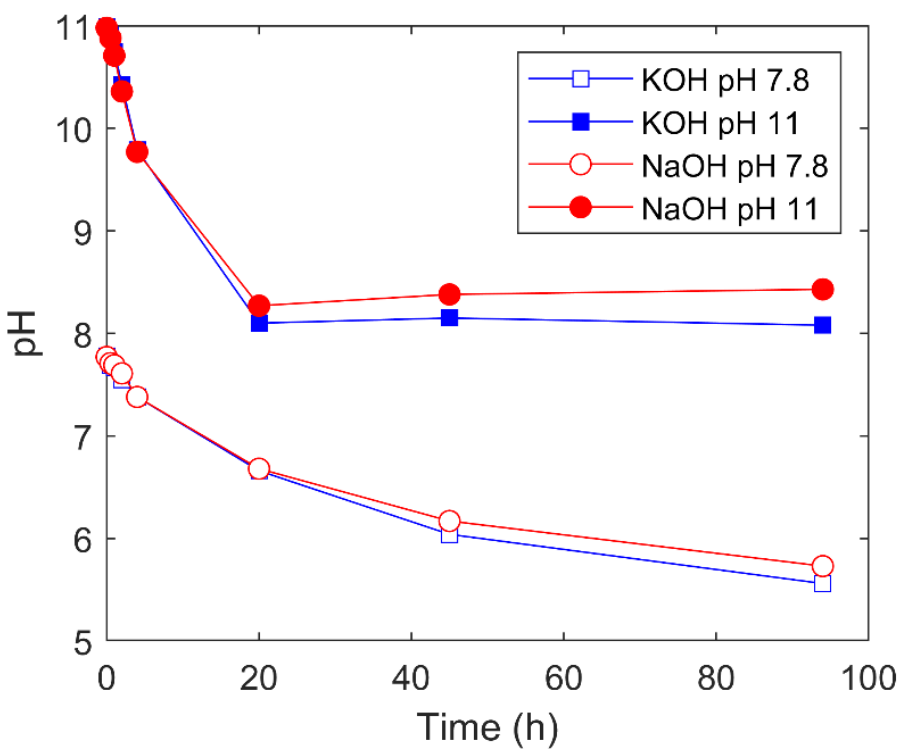

Fig. S1: Time dependence of $\mathrm{pH}$ in reactions using 2 wt\% tannic acid involving $\mathrm{KOH}$ and $\mathrm{NaOH}$ as base with the initial $\mathrm{pH}=7.8$ and 11 .
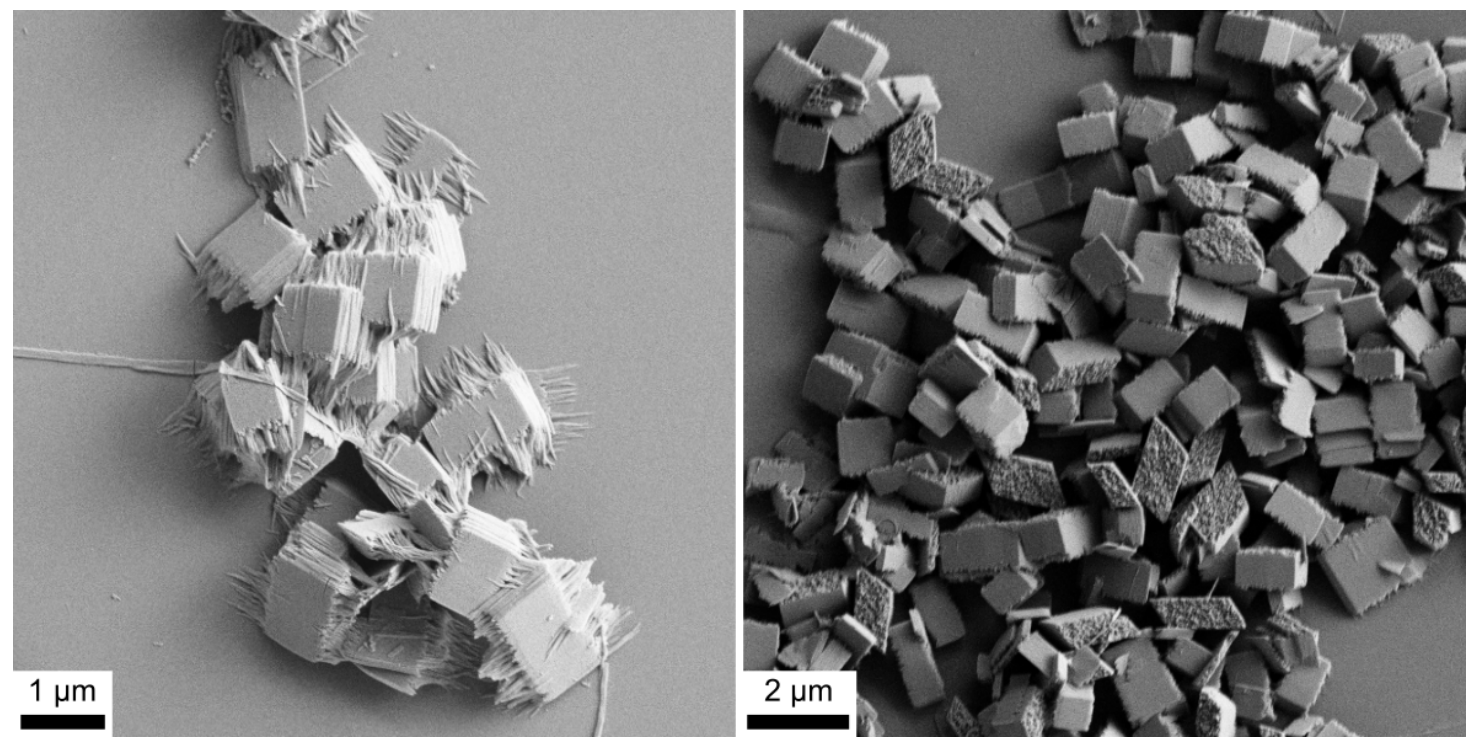

Fig. S2: SEM images of oxidized tannic acid (OTA-KOH) precipitates after $14 \mathrm{~h}$ in $2 \mathrm{wt} \%$ TA solution at initial $\mathrm{pH}=11$ after washing-induced disintegration. 

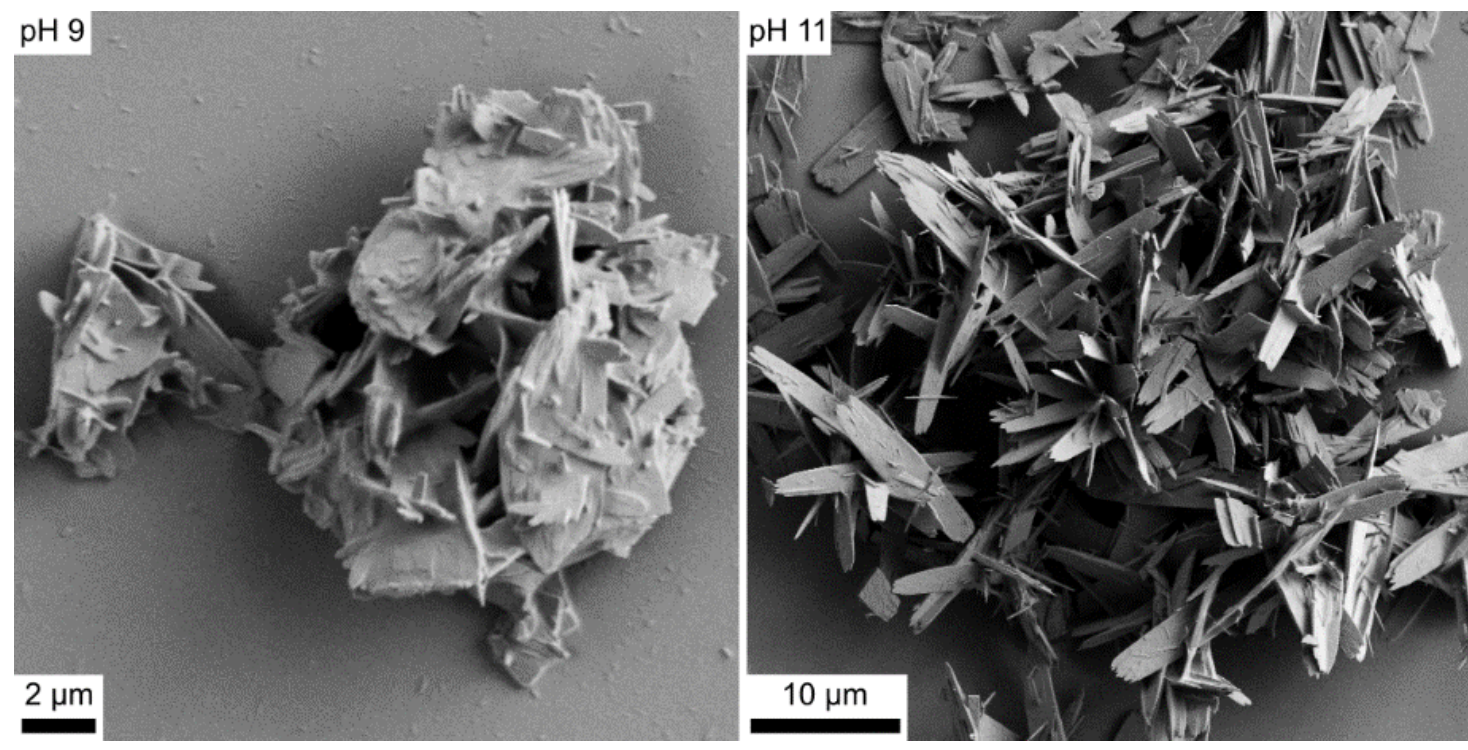

Fig. S3: SEM images of oxidized tannic acid (OTA-NaOH) precipitates after $14 \mathrm{~h}$ in $2 \mathrm{wt} \%$ TA solutions at the given initial $\mathrm{pH}$ (top left corner).

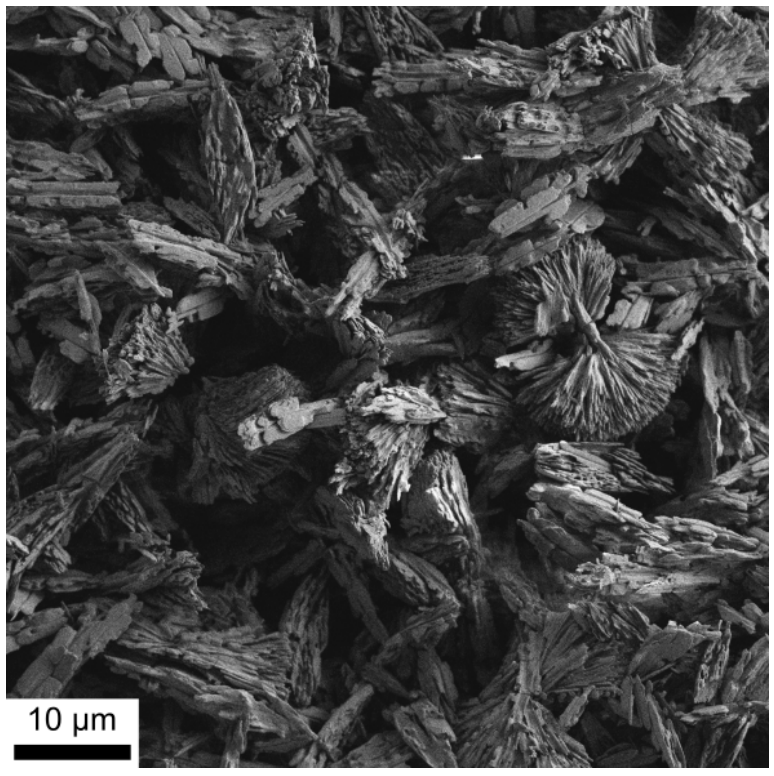

Fig. S4: SEM image of oxidized tannic acid (OTA-LiOH) precipitates after 1 week in 2 wt\% TA solutions at the initial $\mathrm{pH}=11$. 

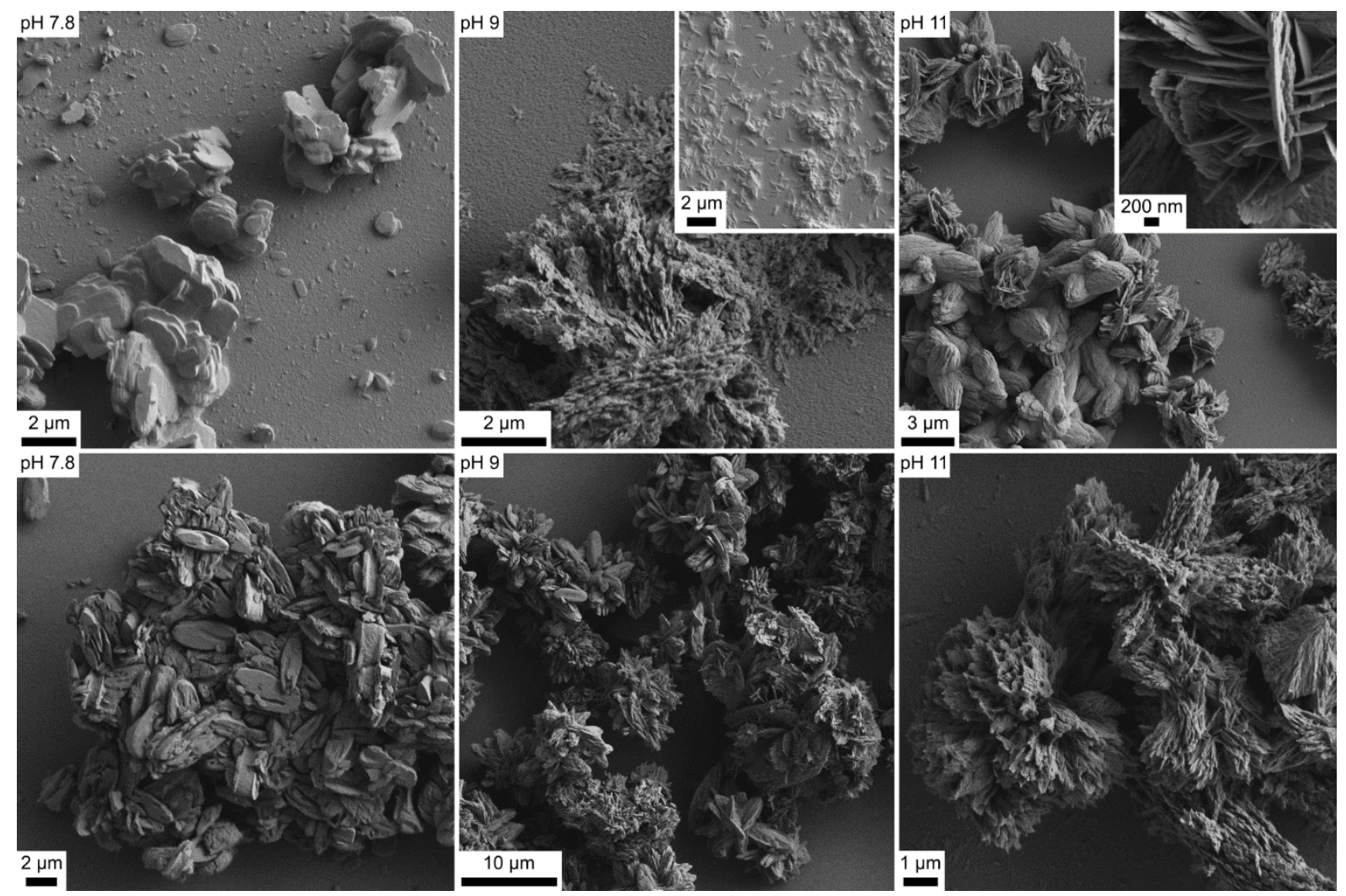

Fig. S5: SEM images of oxidized tannic acid (OTA-Na3 $\mathrm{PO}_{4}$ ) precipitates after $14 \mathrm{~h}$ (top row) and 1 week (bottom row) in $2 \mathrm{wt} \%$ TA solutions at the given initial pH (top left corner).

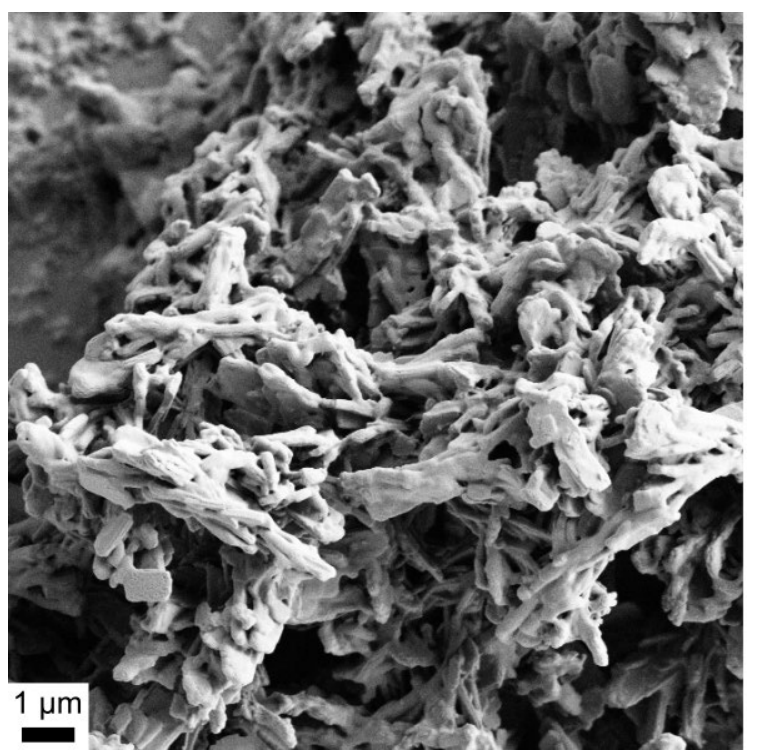

Fig. S6: SEM image of oxidized tannic acid (OTA- $\mathrm{NH}_{4} \mathrm{OH}$ ) precipitates after $14 \mathrm{~h}$ in $2 \mathrm{wt} \%$ TA solutions at the initial $\mathrm{pH}=7.8$. 


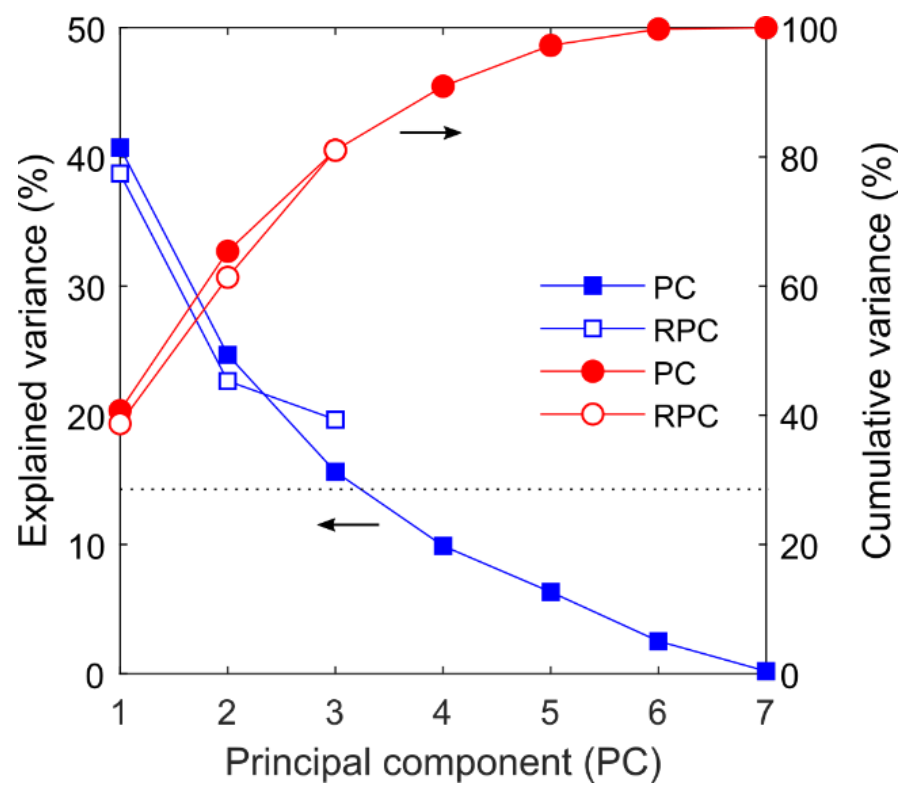

Fig. S7: Explained and cumulative variance of principal components (PCs) and the three varimax-rotated principal components (RPC) used in the analysis. The dotted line depicts unit explained variance (variance was normalized).

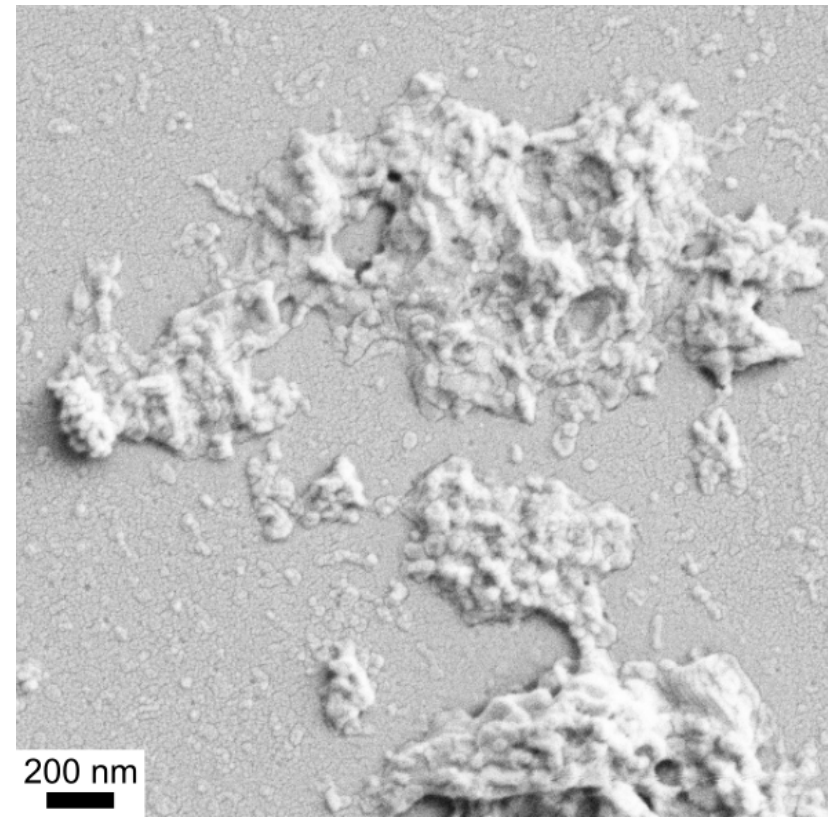

Fig. S8: SEM image of oxidized tannic acid (OTA-NaOH) precipitates after 1 week in $0.2 \mathrm{wt} \%$ tannic acid solution at initial $\mathrm{pH}=7.8$. 

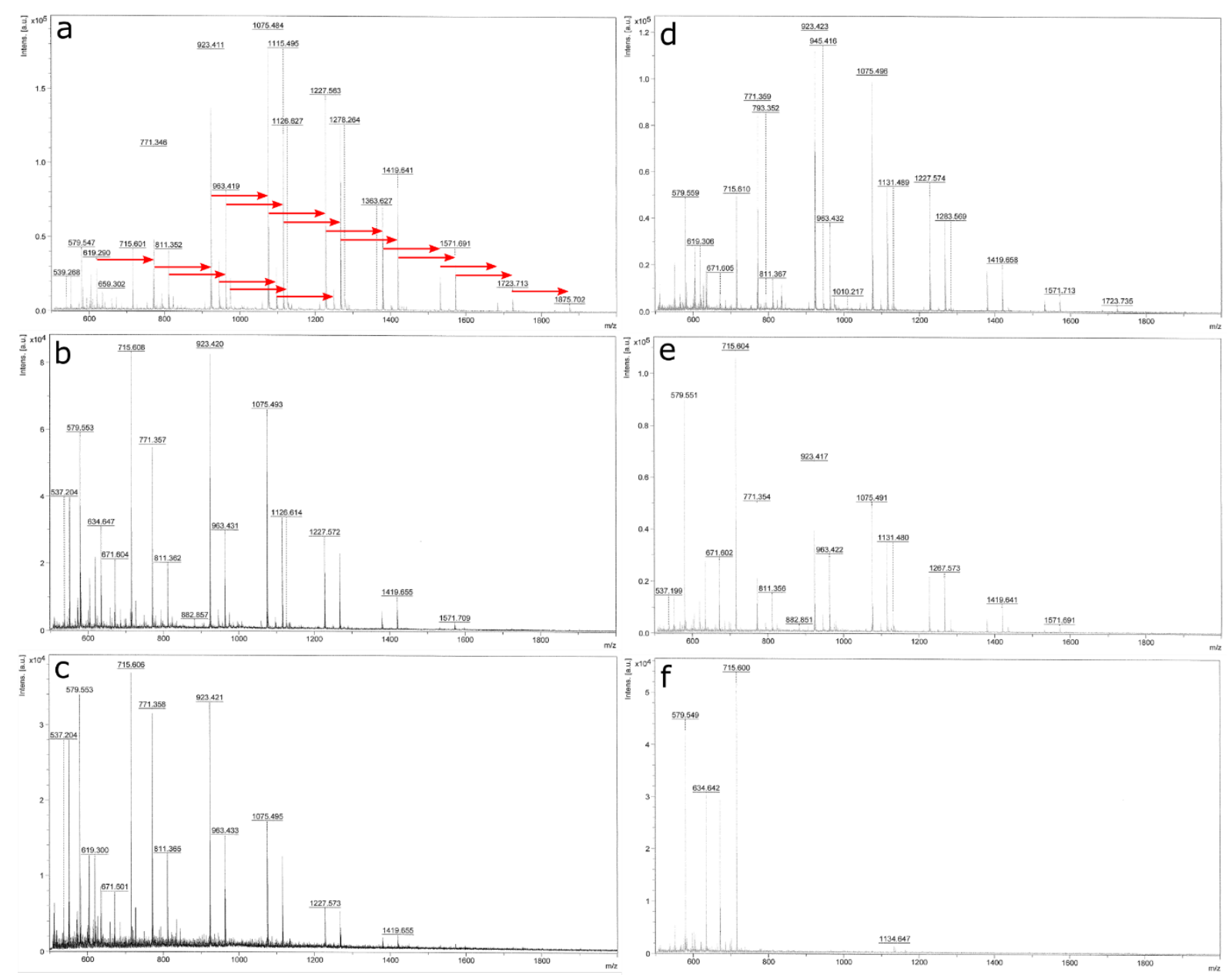

Fig. S9: Matrix-assisted laser desorption ionization (MALDI) mass spectra of oxidized tannic acid (a-c) OTA-NaOH and (d-f) OTA-KOH precipitates after $14 \mathrm{~h}$ at initial pH $(a, d) 7.8,(b, e) 9$ and $(c, f) 11$. The red arrows in (a) correspond to $152 \mathrm{~m} / \mathrm{z}$ (galloyl). Other spectra show similar inter-peak distances. 


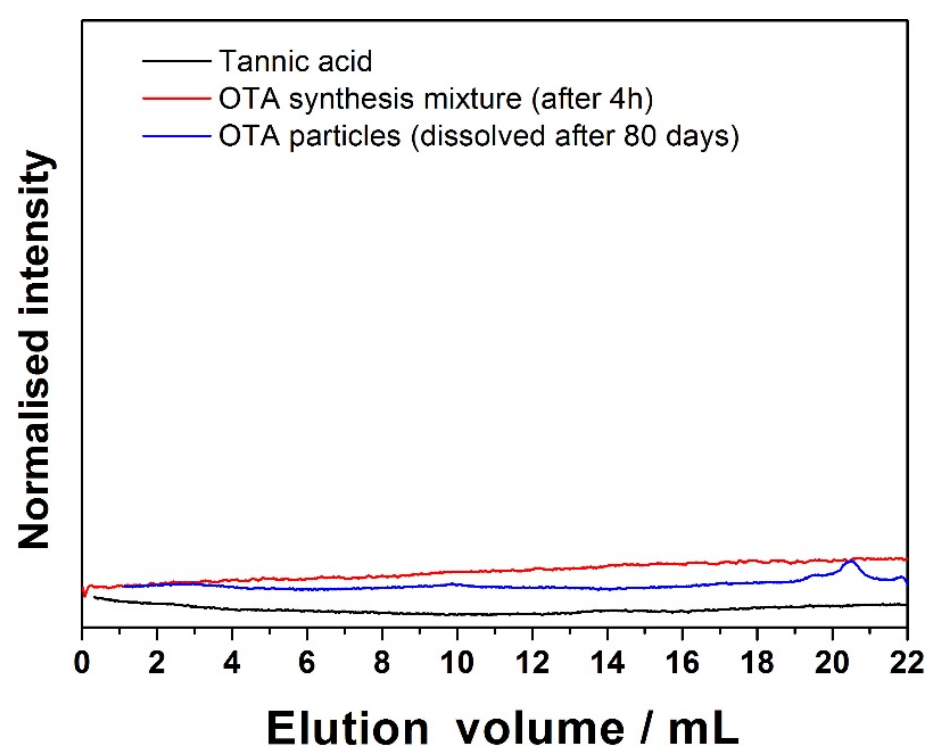

Fig. S10: Gel permeation chromatography elugram of pristine tannic acid, OTA-KOH synthesis mixture at $4 \mathrm{~h}$ and OTA-KOH particles partially dissolved in DMAc 80 days after the synthesis.

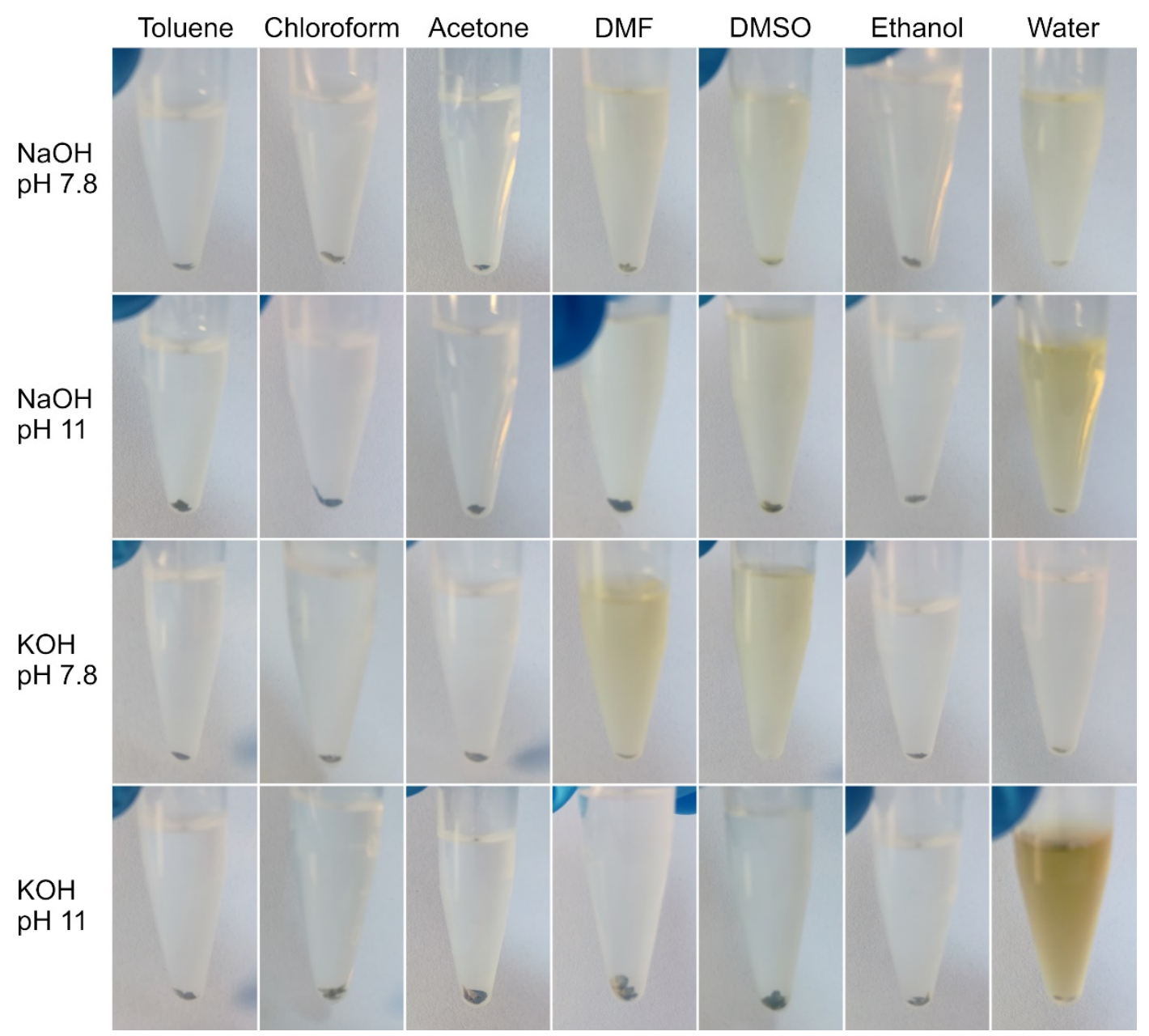

Fig. S11: OTA-NaOH/KOH particle (initial pH 7.8 and 11) qualitative solubility test. Images show the supernatant and sediment after vortex shaking and centrifugation. 


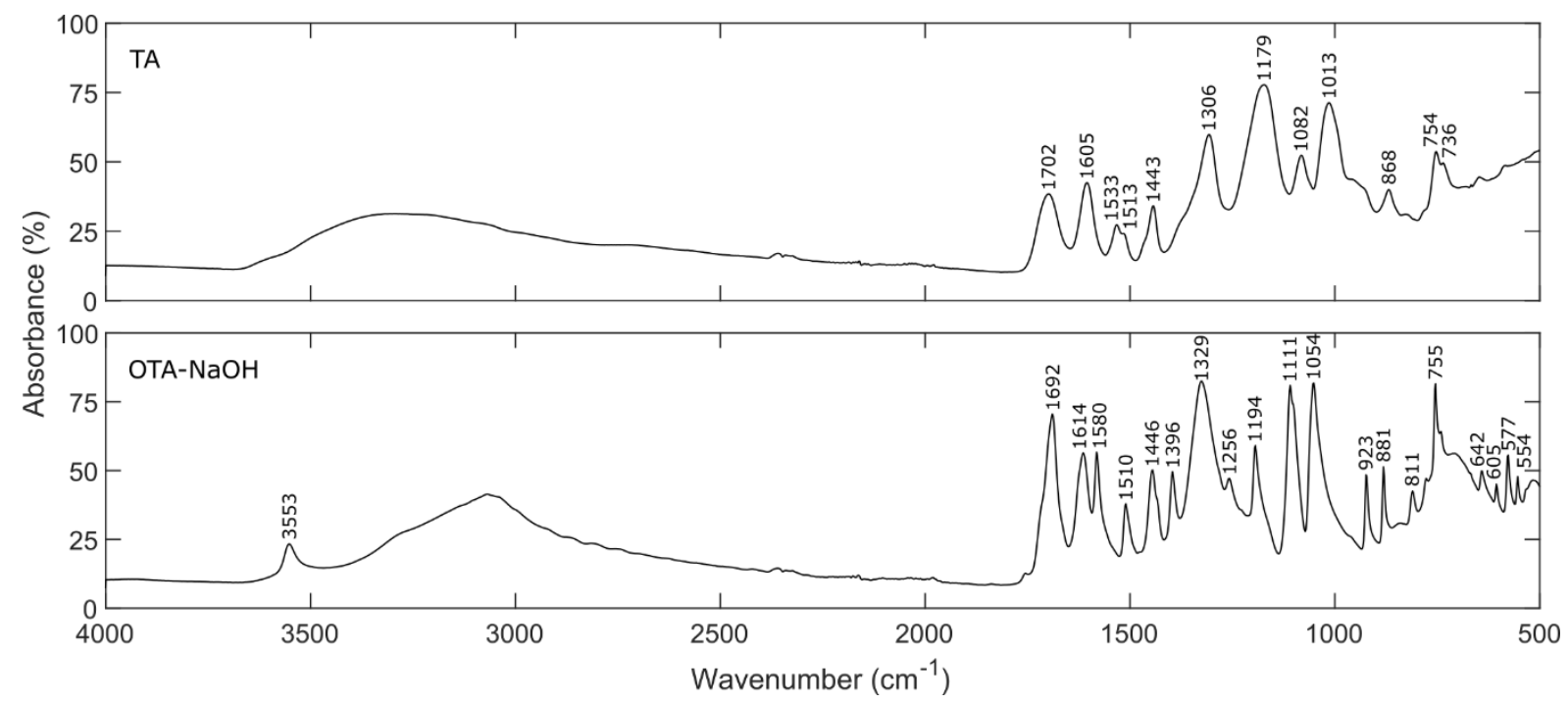

Fig. S12: Infrared spectra of tannic acid (TA) and oxidized tannic acid (OTA-NaOH) precipitates after $14 \mathrm{~h}$ in $2 \mathrm{wt} \%$ TA solution at initial $\mathrm{pH}=7.8$.
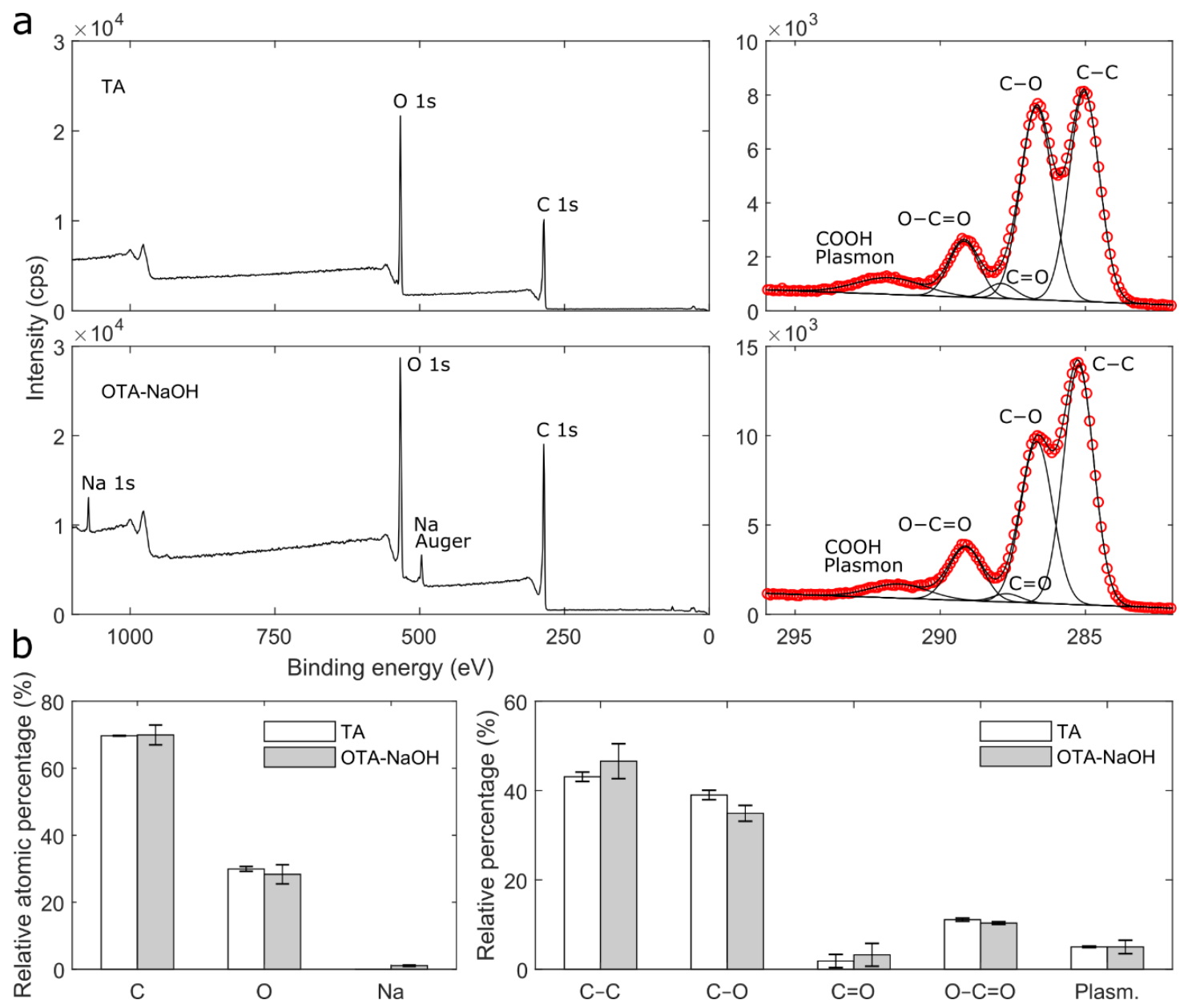

Fig. S13: (a) X-ray photoelectron survey and carbon (C1s) high-resolution spectra of tannic acid (TA) and oxidized tannic acid (OTA- $\mathrm{NaOH}$ ) precipitates collected after $14 \mathrm{~h}$ from $2 \mathrm{wt} \%$ TA solution at initial $\mathrm{pH}=7.8$. (b) Relative atomic percentage of carbon, oxygen and sodium determined from the survey spectra and the relative percentage of carbon bonds determined from the deconvoluted highresolution carbon spectra. The error bars indicate standard deviation. 


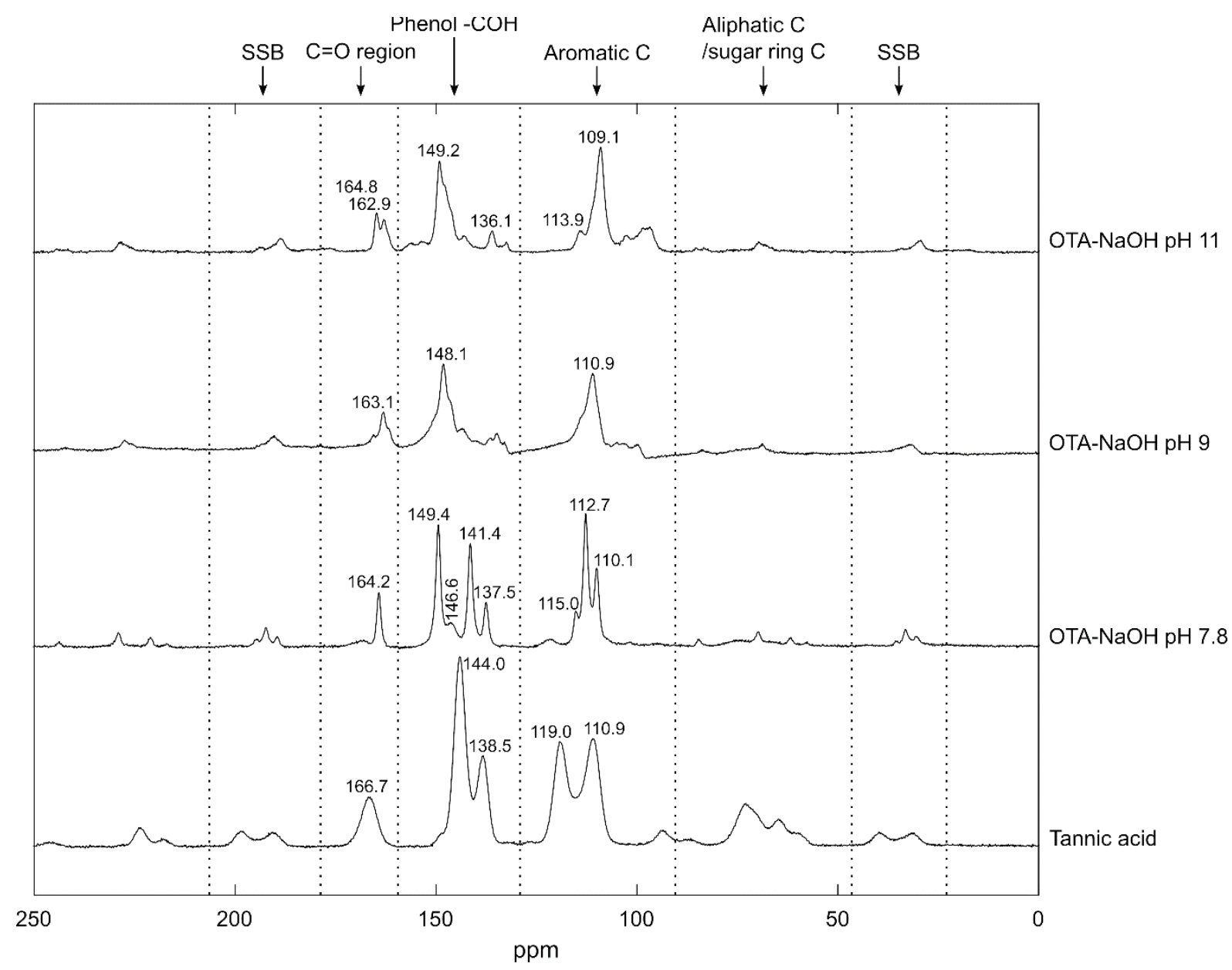

Fig. S14: ${ }^{13} \mathrm{CCP} / \mathrm{MAS}$ NMR spectra of tannic acid and OTA-NaOH particles prepared starting with different initial $\mathrm{pH}$. In the band assignment SSB corresponds to a spinning side band.

\section{References}

1 M. D. Abràmoff, P. J. Magalhães and S. J. Ram, Biophotonics Int., 2004, 11, 36-41.

2 H. Abdi and L. J. Williams, Wiley Interdiscip. Rev. Comput. Stat., 2010, 2, 433-459.

3 D. R. Lide, CRC Handbook of Chemistry and Physics, 96th Edition, 2014.

4 V. A. Pokrovskii and H. C. Helgeson, Chem. Geol., 1997, 137, 221-242.

5 E. K. Barbara Kanegsberg, Handbook for Critical Cleaning: Cleaning Agents and Systems, Second Edition, CRC Press, 2017.

6 D. Beamson, G. Briggs, High Resolution XPS of Organic Polymers: The Scienta ESCA300 Database, 1993, vol. 70. 